\title{
Some concerns regarding the CAT16 chromatic adaptation transform
}

\author{
KEVIN A.G. SMET, ${ }^{1,}{ }^{*}$ ShINING MA, ${ }^{1}$ \\ ${ }^{1}$ ESAT/Light\&Lighting Laboratory, KU Leuven, Ghent 9000, Belgium \\ *Corresponding author: kevin.smet@kuleuven.be
}

Received XX Month XXXX; revised XX Month, XXXX; accepted XX Month XXXX; posted XX Month XXXX (Doc. ID XXXXX); published XX Month XXXX

In recent literature ${ }^{1,2}$, a new chromatic adaptation transform (CAT16) has been published to improve upon the widely used CAT02 model ${ }^{3,4}$. The CAT16 model is based on the form of the CAT02 transform adopted in CIECAM02 ${ }^{3}$, but uses a slightly different sensor space to fix some gamut problems plaguing CIECAM02 and adopts a two-step CAT (as proposed by Smet et al. ${ }^{5}$ ) to ensure symmetry and transitivity ${ }^{2}$. CAT16 is included in CAM16, but is also being promoted as a stand-alone CAT, one that can be used outside of the CAM16 model $^{1}$. However, the use of CAT16, as defined in ${ }^{1}$ and ${ }^{2}$, as a stand-alone model can cause inconsistencies in the calculated corresponding colors due to the presence of the relative luminance of the adapting white $\left(Y_{w}\right)$ in the von Kries-Ives ${ }^{3}$ gain control factors. Such inconsistencies are not present for the standalone version of the CAT02 model $^{4}$, which, unlike the version adopted in CIECAM02, does not include the $\boldsymbol{Y}_{w}$ factors. CAT16 should therefore be modified by omitting the $Y_{w}$ factors. In this paper, we will briefly discuss these issues in more detail and provide a consistent two-step CAT adopting the CAT16 sensor space.

Keywords: CAT16, chromatic adaptation, chromatic adaptation transform, two-step CAT

First, we would like to point out that the concerns raised here don't deny the valuable points of CAT16, but provide insights and recommendations for a more consistent and general implementation.

The von Kries coefficient rule. Chromatic adaptation, the ability of the visual system to adapt to the color of the illumination, is typically modelled, in a first approximation, by the von Kries coefficient rule 6 . The von Kries rule states that the cone sensitivity functions are independently scaled. In other words, to each cone channel a separate gain (multiplicative) control factor is applied:
$\left(\begin{array}{l}R_{0} \\ G_{0} \\ B_{0}\end{array}\right)=\left(\begin{array}{llll}k_{R} & & \\ & k_{G} & \\ & & k_{B}\end{array}\right)\left(\begin{array}{l}R_{a} \\ G_{a} \\ B_{a}\end{array}\right)$

with $R, G$ and $B$ the long, medium and short wavelength cone responses; $k_{R}, k_{G}, k_{B}$ the cone gain control factors; and with subscripts 0 and $a$ denoting baseline and adapted cone signals, respectively.

Von Kries did not specify how the gain control factors $k_{R}, k_{G}, k_{B}$ are to be calculated. However, a common approach, referred to as the von Kries - Ives model, effectively renormalizes the adapted cone responses to an illuminantindependent baseline state using the illuminant color ${ }^{7}$. The $k_{R}, k_{G}, k_{B}$ gain control factors for the Ives model are:

$k_{R}=1 / R_{w} ; k_{G}=1 / G_{w} ; k_{B}=1 / B_{w}$

whereby subscript $w$ refers to the illuminant white point. Although von Kries intended the coefficient rule to apply to cone signals, other 'sensors', such as the ones in the CAT02 ${ }^{4}$

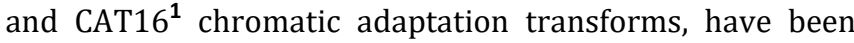
adopted as well, because of their ability to better account for corresponding color (CC) data.

Issues with the CAT16 transform. Following the nomenclature in 2 , the CAT16 chromatic adaptation transform from illuminant $\beta$ to $\gamma$ can be expressed, in its onestep form, as a $3 \times 3$ matrix $\Phi_{\gamma, \beta}$, defined below (Eq. 1 in $^{2}$ ):

$\Phi_{\gamma, \beta}=M_{16}^{-1} \Lambda_{\gamma, \beta} M_{16}$

Where $\beta$ and $\gamma$ represent two different illuminants, $M_{16}$ is the new sensor matrix that transforms tristimulus values XYZ to cone-like sensor responses, RGB. The use of the $M_{16}$ sensor space solves several of the mathematical (gamut) problems plaguing CIECAM02 (see ${ }^{1}$ for a comprehensive overview) and has been shown to lead to similar predictions of corresponding colors than some other proposed sensor spaces, such as CAT02 and HPE ${ }^{1,8}$. 
$\Lambda_{\gamma, \beta}$ is defined as below (Eq. 2 in $^{2}$ ):

$\Lambda_{\gamma, \beta}=\left(\begin{array}{l}D \frac{Y_{w, \beta}}{Y_{w, \gamma}} \frac{R_{w, \gamma}}{R_{w, \beta}}+1-D \\ \end{array}\right.$

$$
D \frac{Y_{w, \beta}}{Y_{w, \gamma}} \frac{G_{w, \gamma}}{G_{w, \beta}}+1-D
$$$$
D \frac{Y_{w, \beta}}{Y_{w, \gamma}} \frac{B_{w, \gamma}}{B_{w, \beta}}+1-D
$$

with $Y_{w, x}$ and $\left(R_{w, x}, G_{w, x}, B_{w, x}\right)$ respectively denoting the $Y$ tristimulus value (or relative luminance to a perfectly reflecting white diffuser) and the sensor responses of the adopted whites under illuminant $x$ ( $=\gamma$ and $\beta$ ), and with $D$ the degree of adaptation. However, as mentioned by Smet et al. ${ }^{5}$, "the interpretation of the degree of adaptation $D_{A, B}$ in one-step transforms is quite unclear: does it correspond to the adaptation level to condition $A$ or $B$, or to some mixed condition $A B$ ? The difficulty arises, because the one-step CAT ignores that each transform from each condition to the illuminant independent baseline state is subject to its own degree of adaptation." Smet et al. ${ }^{5}$ therefore proposed that a more appropriate approach, one that can account for their CC data, is to calculate corresponding colors using a two-step CAT, which passes through an intermediate baseline (perceptually neutral) state using two one-step transforms. Note that the idea of a two-step CAT had also been discussed by other authors, such as Fairchild ${ }^{9}$, Brill ${ }^{10}$ and Hunt ${ }^{11}$.

Although similar predictive performance has been reported for one-step and two-step CATs for the CC data sets used to derive the CAT02 sensor matrix (deviations in CIELAB color difference units are typically less than or equal to 0.1 for most sets, with a maximum of 0.5$)^{2,8}$, improved performance, of up to 1.2 CIELAB units, has been found for the two-step CAT for CC data sets derived using memory color matching, especially for conditions involving colored illuminants ${ }^{12}$. Furthermore, the two-step transform is symmetrical and transitive, even when $\mathrm{D} \neq 1$, while the one-step CAT is not ${ }^{1}$.

Li et al. ${ }^{2}$ adopted such a two-step approach as follows:

$$
\Pi_{\gamma, \beta}=M_{16}^{-1}\left(\Lambda_{E, \gamma}\right)^{-1} \Lambda_{E, \beta} M_{16}
$$

whereby the equi-energy white (illuminant E) has been adopted as the intermediate baseline state.

Indeed, Smet et al. ${ }^{5}$ wrote "To be able to serve as a reference for more or less chromatic illumination conditions under incomplete adaptation, the illuminant independent baseline state is typically assumed to correspond to the neutral (achromatic) point, i.e. the stimulus that looks neither red, yellow, green or blue, under dark adapted conditions. The equienergy-white (EEW) is therefore. often adopted as the intrinsic white point in many chromatic adaptation transforms and color appearance models. However, it should be noted that the observer variability under dark adapted conditions is quite large and several other unique white (neutral) chromaticities have been reported."

Since for illuminant E, $R_{w}=G_{w}=B_{w}=X_{w}=Y_{w}=Z_{w}$, Li et al. ${ }^{2}$ used the following formulation for $\Lambda_{E, \beta}$ (Eq. $19 \mathrm{in}^{2}$ ):

$$
\Lambda_{E, \beta}=\left(\begin{array}{lll}
D \frac{Y_{w, \beta}}{R_{w, \beta}}+1-D & & \\
& D \frac{Y_{w, \beta}}{G_{w, \beta}}+1-D & \\
& & D \frac{Y_{w, \beta}}{B_{w, \beta}}+1-D
\end{array}\right)
$$

with $D$ the degree of adaptation going from illuminant $\beta$ to illuminant E. $\Lambda_{E, \gamma}$ can be defined analogously.

However, this definition of the CAT16 transform can provide inconsistent corresponding colors when used as a standalone CAT. This is easy to see when considering the prediction of the adapted white under full adaptation $(D=1)$ to illuminants $\beta$ and $\gamma$. In this case, we expect to have the white point under illuminant $\gamma$ as corresponding color of the white under illuminant $\beta$. However, after setting $D=1$ and multiplication of the two diagonal matrices, Eq. (5) results in:

$$
\Pi_{\gamma, \beta}=M_{16}^{-1}\left(\begin{array}{lll}
\frac{Y_{w, \beta}}{Y_{w, \gamma} \frac{R_{w, \gamma}}{R_{w, \beta}}} & \\
& \frac{Y_{w, \beta} \frac{G_{w, \gamma}}{Y_{w, \gamma}}}{G_{w, \beta}} & \\
& \frac{Y_{w, \beta}}{Y_{w, \gamma}} \frac{B_{w, \gamma}}{B_{w, \beta}}
\end{array}\right) M_{16}
$$

The corresponding cone responses $\left(R_{w, \beta, c}, G_{w, \beta, c}, B_{w, \beta, c}\right)$ of the white point under illuminant $\beta\left(R_{w, \beta}, G_{w, \beta}, B_{w, \beta}\right)$ can therefore only be equal to those of the white point under illuminant $\gamma$ $\left(R_{w, \gamma}, G_{w, \gamma}, B_{w, \gamma}\right)$ when the (relative) luminance values under the two illuminants are equal, i.e. when $Y_{w, \beta} / Y_{w, \gamma}=1$; if $Y_{w, \beta} \neq Y_{w, \gamma}$, the white point under illuminant $\beta$ cannot be mapped to that under illuminant $\gamma$, as is clear from the following equality:

$$
\begin{aligned}
& \left(\begin{array}{l}
R_{w, \beta, c} \\
G_{w, \beta, c} \\
B_{w, \beta, c}
\end{array}\right)=\frac{Y_{w, \beta}}{Y_{w, \gamma}}\left(\begin{array}{ccc}
R_{w, \gamma} / R_{w, \beta} & & \\
& G_{w, \gamma} / G_{w, \beta} & \\
& & B_{w, \gamma} / B_{w, \beta}
\end{array}\right)\left(\begin{array}{l}
R_{w, \beta} \\
G_{w, \beta} \\
B_{w, \beta}
\end{array}\right) \\
& \left(\begin{array}{l}
R_{w, \beta, c} \\
G_{w, \beta, c} \\
B_{w, \beta, c}
\end{array}\right)=\frac{Y_{w, \beta}}{Y_{w, \gamma}}\left(\begin{array}{l}
R_{w, \gamma} \\
G_{w, \gamma} \\
B_{w, \gamma}
\end{array}\right)
\end{aligned}
$$

The $Y_{w}$ factor was originally introduced in CIECAM02 "to ensure that the adaptation is independent of the luminance factor of the adopted white point"13, or in other words, to ensure that the effect of the CAT is "limited to changing only on the chromaticity of the illuminant"14. The result of such a transform is that it generates corresponding colors with the same (relative) luminance values as the original colors. Whether such luminance constancy is a desired property of a CAT is debatable, as it distorts the relative magnitude of sensor responses of the corresponding colors to those of the adopted white point under the second illumination.

For example, let's consider a set of neutral greys with $Y$ values ranging from 10 to 50 under illumination condition $\beta$ with the white point having $Y_{w, \beta}=60$ (the actual value doesn't matter, but is chosen small to better illustrate the effect in Fig. 1) and their corresponding colors under an illumination 
$\gamma$, which has the same chromaticity as $\beta$, but a (relative) luminance of $Y_{w, \gamma}=90$ (for simplicity we set $D=1$ ). Because of the inclusion of the $Y_{w}$ factors, the relative differences in the sensor responses of the white points under the two illumination conditions cancel out (see Eq. 7) - as intended by the original CAT16 model. The calculated corresponding colors of these greys under illumination $\gamma$ will now be identical, in both chromaticity and (relative) luminance (see red dotted vertical lines in Fig. 1), to the original stimuli under illumination condition $\beta$. However, the assumed white point under illumination $\gamma$ has $Y_{w, \gamma}=90$, not 60 as predicted. Note that a stimulus with $Y=60$ would actually have a greyish appearance under an illuminant $\gamma$ where the white is considered to have a $Y=90$. Therefore, contrary to the concept of corresponding colors (same color appearance under two illumination conditions), the original CAT16 transform maps the original greys to a set of 'corresponding' greys with different lightness values (e.g. CIELAB $L^{*}$ ), as can be seen in Fig. 1. Note that a von Kries type CAT without $Y_{w}$ factors, retains the lightness values (horizontal green dotted lines in Fig. 1) and is thus better suited to map between colors with the same appearance.

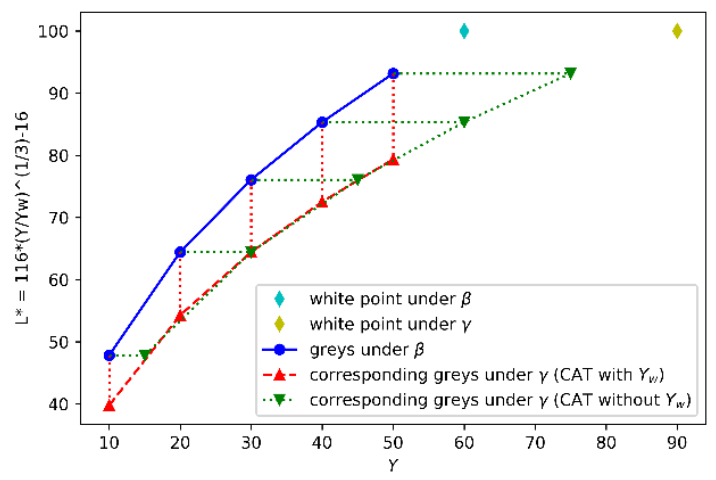

Fig. 1. Illustration of possible lightness distortion caused by a CAT transform that includes $Y_{w}$ factors. Yellow and cyan diamonds represent the white point under illuminations $\beta$ and $\gamma$, respectively. Blue points, with $Y$ values ranging from 10 to 50, represent the set of original greys under illumination $\beta$. Red triangles, with the same $Y$ value as the blue points, represent the 'corresponding' greys from illumination $\gamma$ to $\beta$, calculated using CAT16 (with $Y_{w}$ factors, Eq. 7) when $D=1$. The green triangles, with the same lightness value as blue points, represent the corresponding greys from illumination $\gamma$ to $\beta$ calculated by the CAT (without $Y_{w}$ factors) proposed in Eq. (9).

As suggested by the above simple examples, and as already stated in a special section on this issue in Fairchild's book on Color Appearance Models (see p. 268-269 in ${ }^{15}$ or p. 291-292 in $\left.{ }^{16}\right)$, the inclusion of the $Y_{w}$ factors can lead to inconsistent corresponding color predictions. According to Fairchild, the $Y_{w}$ factors are "a remnant of an earlier model formulation

\footnotetext{
1 Note that when a sensor space is used that is normalized to illuminant E, i.e. $X_{w, E}=R_{E}=G_{E}=B_{E}=1, \Lambda_{\mathrm{A} \rightarrow \mathrm{E}}=1 / \mathrm{X}_{w, A}$ is equal to the gain control factors proposed by Ives ${ }^{7}$ (see Eq. 2). The choice of using the reciprocal responses of the adopted white as gain control
}

(similar to that in CIECAM97s) that was not corrected prior to publication of the CIE technical report."15 and he mentions that a transform that includes them "cannot be used without the remainder of the CIECAM02 model." 15.

Repairing the CAT16 transform form. Because of the possible inconsistent corresponding color predictions of the CAT16 model defined in ${ }^{1}$ and ${ }^{2}$, we therefore propose that the $Y_{w}$ factors be removed from the model when no explicit luminance constancy is required (Eq. 9):

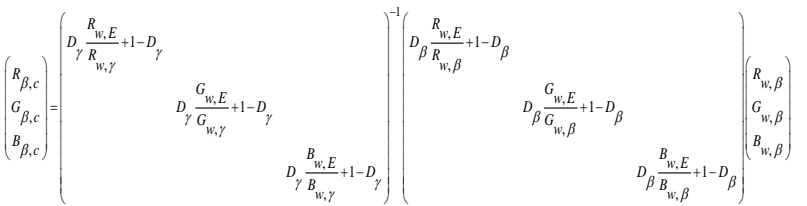

This two-step transform is identical to the more general twostep transform already proposed in Eq. 12 in Smet et al.5:

$X_{B}^{\prime}=\left(D_{B, 0} \cdot \Lambda_{B \rightarrow 0}+\left(1-D_{B, 0}\right)\right)^{-1} \cdot\left(D_{A, 0} \cdot \Lambda_{A \rightarrow 0}+\left(1-D_{A, 0}\right)\right) \cdot X_{A}$

with, in the CAT16 case, $X_{A}$ a vector containing the three responses $(R, G, B)$ of a stimulus under illumination $A$ represented in the $\mathrm{M}_{16}$ sensor space, $\Lambda_{\mathrm{A} \rightarrow 0}=X_{w, 0} / X_{w, A}$ the ratio of the sensor responses of the baseline illuminant $E$ (assumed to be perceptually neutral) to those of the adopted white $A$, and with $D_{A, 0}$ denoting the degree of adaptation under illumination $A$ (with $\mathrm{E}$ as baseline!). Similar definitions apply to $X_{B}, \Lambda_{\mathrm{B} \rightarrow 0}$ and $D_{B, 0}$ for illumination $B$.

Because the model does not include the $Y_{w}$ factor of the white point, it is also more easily applicable to situations for which these are difficult or impossible to define, such e.g. as uncorrelated self-luminous stimuli characterized only by their absolute tristimulus values or spectral radiances, as in e.g. ${ }^{17}$.

Finally, note that in its most general form, the two-step model in Eq. 10 (or Eq. 12 in ${ }^{5}$ ) only suggests the use of illuminant $\mathrm{E}$ as a potentially viable baseline, but does not actually fixes it to some specific value (i.e. $X_{w, 0}$ are not set to 100 or 1 , depending on how the $X Y Z$ or $R G B$ input is normalized) ${ }^{1}$. The reason is that, in addition to illuminant $E$, several other unique white chromaticities have been proposed in literature and that the baseline could therefore perhaps be further improved upon in future research. For example, Smet et al. ${ }^{18}$ reported that under different luminance levels, the unique white in object mode is centered at approximately $6000 \mathrm{~K}$ and located below the blackbody locus, while the unique white in illuminant mode ${ }^{19}$ is approximately centered around $6500 \mathrm{~K}$, which is slighter higher than that in object mode. Zhai et al. ${ }^{20}$ conducted a neutral white matching experiment in a wide range of different adapting illuminants using two kind of media, surface and self-luminous colors. It has also been found that the baseline illuminant depends on the viewing media. For surface colors, the correlated color

factors can therefore be considered equivalent to an adaptation to an illuminant $E$ baseline. This still holds in case the sensor space is normalized to 100 , as is common practice in color appearance models, because when $X_{W, E}^{\prime}=R_{E}^{\prime}=G_{E}^{\prime}=B_{E}^{\prime}=100$ then $\Lambda_{\mathrm{A} \rightarrow \mathrm{E}}=100 / \mathrm{X}_{w, A}^{\prime}=1 / \mathrm{X}_{w, A}$ 
temperature $(C C T)$ of the baseline illuminant tends to range from $6000 \mathrm{~K}$ to $7000 \mathrm{~K}$ and the Duv - distance to the blackbody locus in the CIE 1960 chromaticity diagram - from 0 to 0.005 , while for self-luminous medium, the CCT of the baseline is around $9000 \mathrm{~K}$ and the Duv ranges from -0.001 to 0.003 . These results indicate that the baseline deviates from illuminant E $(C C T=5455 \mathrm{~K}, D u v=-0.0044)$ towards higher CCT levels and that it is dependent on viewing mode and medium.

Non-linear CATs? Although it is debatable that a mere linear rescaling, such as in Eq. 10, is able to account for differences in luminance levels under two illumination conditions (e.g. to enable lightness constancy), it can be noted that several authors have reported quite good agreement between predicted matches and calculated matches, even under conditions with (not too) different adapting luminance. For example, Bäuml reported that a von Kries type model provided a good, but not perfect, description of the data of an experiment with illuminants varying in chromaticity and luminance of the white point, and with backgrounds varying in average luminance and color ${ }^{21}$, as did Brainard and Wandell (see e.g. the good linearity between predicted and measured matches in terms of $L^{*}, u^{*}, v^{*}$ in Fig. 6 in ${ }^{22}$ ).

However, it should be noted that a stand-alone CAT provides only a first approximation to corresponding colors under different illumination, or more general viewing conditions. Indeed, other authors have for example reported deviations from linearity ${ }^{23}$ or the need for two-stage models that include multiplicative scaling and subtractive terms ${ }^{24}$.

Generally, corresponding colors under the two adapted conditions can be considered to refer to equal perceptual attributes, such as provided by a (non-linear) Color Appearance Model (CAM), instead of equal sensor responses provided by a (linear) von Kries type CAT (without $Y_{w}$ factors).

Nevertheless, from a practical point of view, simple, linear, stand-alone CATs remain a useful tool to account for not-toodifferent illumination conditions. For example, to map to corresponding tristimulus values under the assumed white point of some color space, such as D65 for CIELAB.

For an overview of the various components and stages of chromatic adaptation (transform), including non-linear response compression, the reader is also referred to ${ }^{5}$.

Conclusions. The two-step CAT16 model as defined in ${ }^{1,2}$, can result in inconsistent corresponding colors when adopted independently from CAM16, due to the inclusion of the $Y_{w}$ factors of the adopted whites. Although in certain cases, when explicit luminance constancy is required, such a model could be useful. Additionally, the fixed illuminant $E$ baseline in CAT16 might not be appropriate for all conditions. However, the change from the $\mathrm{M}_{02}$ sensor space adopted in CAT02 to the new $\mathrm{M}_{16}$ sensor space does correct the gamut problems plaguing CIECAM02, while keeping similar predictive performance of the $\mathrm{CAT}^{1,2}$. A more consistent two-step CAT16 model, as defined in Eq. 10 is therefore proposed for stand-alone use or inclusion in CAMs.

Disclosures. The authors declare no conflicts of interest.

\section{References}

1. Li C, Li Z, Wang Z, et al. Comprehensive color solutions: CAM16, CAT16, and CAM16-UCS. Color Res Appl. 2017:1-16. doi:10.1002/col.22131

2. Li C, Xu Y, Wang Z, et al. Comparing two-step and one-step chromatic adaptation transforms using the CAT16 model. Color Res Appl. 2018;43(5):633-642. doi:10.1002/col.22226

3. CIE159-2004. A Colour Apperance Model for Color Management System: CIECAM02. Vol CIE159-200. (Report CIET-01 T, ed.). Vienna: $\mathrm{CIE} ; 2004$.

4. CIE16x-2004. A Review of Chromatic Adaptation Transforms. Vol CIE160-200. Vienna: CIE; 2004.

5. Smet KAG, Zhai $Q$, Luo MR, Hanselaer $P$. Study of chromatic adaptation using memory color matches, Part I: neutral illuminants. Opt Express. 2017;25(7):7732-7748. doi:10.1364/OE.25.007732

6. von Kries J. Influence of adaptation on the effects produced by luminous stimuli. MacAdam DL, ed. 1905; Sources of.

7. Ives HE. The relation between the color of the illuminant and the color of the illuminated object. Trans Illum Eng Soc. 1912;7:11.

8. Derhak M, Graphics O. Color appearance processing using iccMAX. Electron Imaging. 2018;2018(16):323-1-323-326.

9. Fairchild MD. Formulation and testing of an incomplete-chromaticadaptation model. Color Res Appl. 1991;16(4):243-250. doi:10.1002/col.5080160406

10. Brill MH. Demystifying CIECAM97s: A reply to Hunt. Color Res Appl. 1999;24(3):217. 6378(199906)24:3<217::AID-COL10>3.0.CO;2-C

11. Hunt RWG. Some comments on using the CIECAM97s colourappearance model. Color Res Appl. 1999;24(3):214-215. doi:10.1002/(SICI)1520-6378(199906)

12. Smet KAG, Zhai Q, Luo MR, Hanselaer P. Study of chromatic adaptation using memory color matches, Part II: colored illuminants. Opt Express. 2017;25(7):8350-8365. doi:10.1364/OE.25.008350

13. Moroney N, Fairchild MD, Hunt RWG, Li C, Luo MR, Newman T. The CIECAM02 color appearance model. IS\&T/SID Tenth Color Imaging Conf. 2002:23.

14. Hunt RWG, Li C, Luo MR. Chromatic adaptation transforms. Color Res Appl. 2005;30(1):69-71. doi:10.1002/col.20085

15. Fairchild MD. Color Appearance Models. 2nd ed. Chichester: John Wiley \& Sons; 2005.

16. Fairchild MD. Color Appearance Models. 3rd ed. Chichester: John Wiley \& Sons; 2013.

17. Hermans $S$, Smet KAG, Hanselaer P. Color appearance model for selfluminous stimuli. J Opt Soc Am A. 2018;35(12):2000-2009. doi:10.1364/JOSAA.35.002000

18. Smet K, Deconinck G, Hanselaer P. Chromaticity of unique white in object mode. Opt Express. 2014;22(21):25830-25841. https://www.osapublishing.org/oe/abstract.cfm?uri=oe-22-2125830.

19. Smet KAG, Deconinck G, Hanselaer P. Chromaticity of unique white in illumination mode. Opt Express. 2015;23(10):12488-12495. doi:10.1364/OE.23.012488

20. Zhai Q, Luo MR. Study of chromatic adaptation via neutral white matches on different viewing media. Opt Express. 2018;26(6):77247739. doi:10.1364/OE.26.007724

21. Bäuml K-H. Simultaneous color constancy: how surface color perception varies with the illuminant. Vision Res. 1999;39(8):15311550. doi:http://dx.doi.org/10.1016/S0042-6989(98)00192-8

22. Brainard DH, Wandell BA. Asymmetric matching - How color appearance depends on the illumant. J Opt Soc Am a-Optics Image Sci Vis. 1992;9(9):1433-1448. doi:10.1364/josaa.9.001433

23. Lucassen MP, Walraven J. Separate processing of chromatic and achromatic contrast in color constancy. Color Res Appl. 2005;30(3):172-185. doi:10.1002/col.20105

24. Jameson D, Hurvich LM. Color adaptation: Sensitivity control, contrast, after-images. In: D. Jameson \& LMH, ed. Handbook of Sensory Physiology. Berlin: Springer; 1972:568-581. 


\section{Author bios}

Kevin A.G. Smet, obtained his PhD in Engineering at $\mathrm{KU}$ Leuven in 2011. He is currently appointed as an Associate Research Professor at KU Leuven. Kevin is the team leader of the Appearance \& Perception group of the Light\&Lighting Laboratory and is an active member of CIE Division 1. His research interests include color rendition and color quality of white light sources, color appearance modeling, memory and preferred colors, perception of light and color in virtual reality and color science in general.

Shining Ma received her BS and MS in optical engineering from Zhejiang University (2017). Now she is a PhD researcher in ESAT/Light\&Lighting Laboratory, KU Leuven. Her PhD subject is 'Chromatic adaptation in complex scenes'. 